\title{
Towards a Mobility-Supporting Incentive Scheme for Peer-to-Peer Content Distribution
}

\author{
Matthias Wichtlhuber and David Hausheer* \\ Peer-to-Peer Systems Engineering, TU Darmstadt, Germany \\ \{mwichtlh, hausheer\}@ps.tu-darmstadt.de
}

\begin{abstract}
The distribution of bulk content such as video streams and system updates is becoming increasingly important on mobile devices. For this kind of data transfers, the application of the Peer-to-Peer (P2P) paradigm is attractive for content providers, as it allows to leverage clients' (peers') resources for the dissemination process. The contribution of resources can be encouraged by applying reciprocal incentives, which prevent a peer from consuming more resources than he contributed to the system. However, reciprocation has drawbacks, e.g., in terms of high energy consumption and low performance, when applied to mobile peers. This work substantiates these problems through measurements and discusses a virtual node concept as a possible solution.
\end{abstract}

\section{Introduction}

The widespread adoption of smartphones has led to a growing volume of mobile traffic. This increase in volume is driven by a few types of applications generating comparably high data volume, e.g., according to recent studies [1] more than $50 \%$ of mobile traffic is real-time entertainment traffic being caused mainly by audio/video streaming applications. In order to transfer such bulky data, content providers can utilize the Peer-to-Peer (P2P) approach to reduce their costs [2]. A $\mathrm{P} 2 \mathrm{P}$ content distribution system requires clients (peers) to forward parts of the content (chunks) amongst each other, instead of retrieving the entire content from the central server. Thus, the consumption of resources like bandwidth, storage and CPU power is shifted from the content provider's data center to the peers.

The utilization of peers' resources implies the need for incentivation, as peers act rationally in the economic sense, resulting in a behavior that maximizes the service received from the network and minimizes the contribution of own resources. Researchers have proposed a number of incentive schemes to ensure contribution of peers in content distribution, where the most common form of incentive schemes applied today are reciprocal incentive schemes [3] [4] [5] very similar to BitTorrent's tit-for-tat approach. This class of schemes requires peers to provide other peers the same amount of resources, as they consume, e.g.,

\footnotetext{
* This work has been supported in parts by the European Union (FP7/\#317846, SmartenIT and FP7/\#318398, eCousin) and the German DFG (CRC 1053, MAKI).
} 


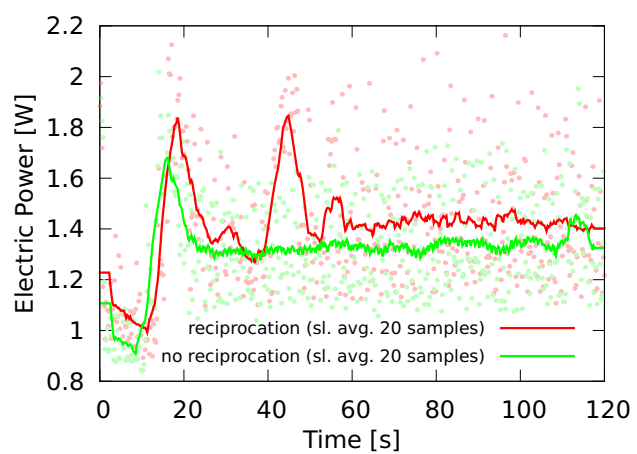

(a) Time Series

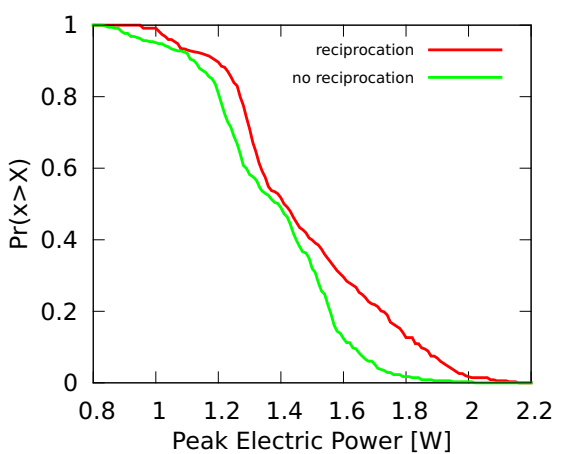

(b) Counter CDF

Fig. 1. Energy measurements comparing reciprocation and pure downloading

in the case of bandwidth, by keeping the ratio between upload and download bandwidth close to 1 .

\section{Problem Description}

Reciprocal schemes show weaknesses in heterogeneous environments, as they discriminate peers with poor resources, i.e., mobile peers. From a mobile user's perspective, reciprocation is an unattractive option, as sending data is more expensive in terms of energy consumption than just receiving data. The penalty of reciprocation can be quantified by measuring the energy consumption of a mobile P2P video on demand streaming application [2] running on an Android device using Wi-Fi (see Figure 1a). After a phase of aggressive scheduling (10s $55 \mathrm{~s}$ ), the measurement shows reciprocation to be about $100 \mathrm{~mW}$ more expensive than just downloading in steady state $(55 \mathrm{~s}-120 \mathrm{~s})$. This gap is caused by high peak values: plotting the counter cumulative distribution function of the peak power consumption (Figure 1b shows a higher probability of high peak values in the case of reciprocation. It is reasonable to assume the gap to broaden with increased bitrates and for link types with a higher transmitting power such as HSDPA and LTE. Besides that, reciprocation is also expensive in the monetary sense for both, user and network provider.

A second field of problems is caused by the inherent properties of mobile links, namely varying data rates and frequent link loss. Trading partners cannot distinguish whether a mobile peer has bad link quality or tries to steal bandwidth. A strict enforcement of reciprocation under varying bandwidth constraints can even lead to a breakdown of cooperation, as partners start reducing service for each other in an alternating fashion [3. Moreover, with current schemes, frequent link loss looks like a peer failure followed by a new peer joining the network. This is problematic, as reciprocal incentive schemes rely on a grace period, in which new peers are served with the lowest possible priority only. Thereby, new peers 


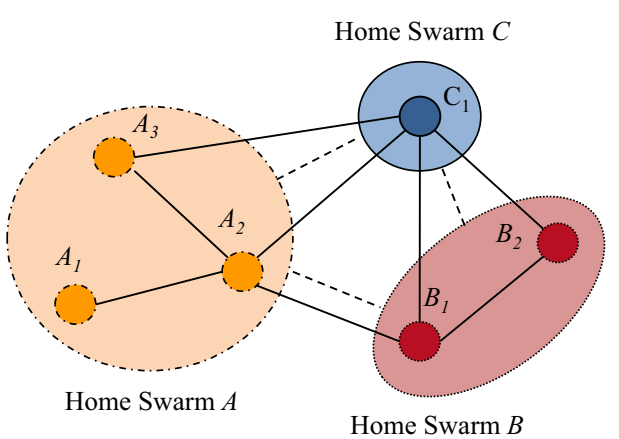

(a)

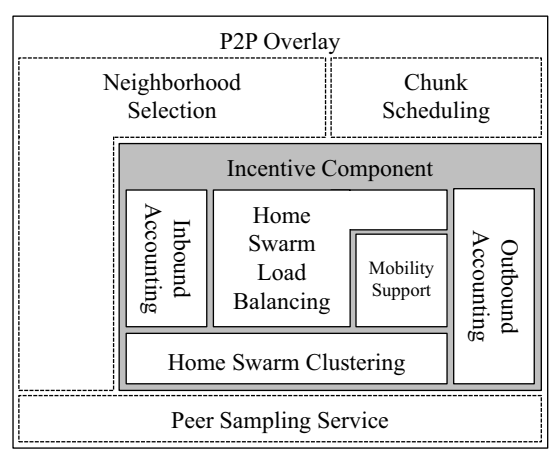

(b)

Fig. 2. Random graph with clustered home swarms and architecture draft

can gain some bargaining power, as they do not have content to reciprocate, while the possibilities to steal resources are kept low. Thus, it takes some time for reciprocal incentive schemes to gain momentum, resulting in a bad perceived quality for mobile users after link failures.

Although reciprocation is problematic with respect to mobility, dropping the incentive scheme for mobile users is not an option. On the one hand, there are practical considerations like the infeasibility of telling apart mobile from wired users by relying on their IP address only. On the other hand, an increased fraction of mobile users in a P2P network not participating in distributing content leads to a service degradation of the complete network, as the overall available upload bandwidth determines the overall available download bandwidth. Thus, there is a need for reciprocal incentive schemes supporting mobility in $\mathrm{P} 2 \mathrm{P}$ content distribution.

\section{Approach and Proposed Architecture}

The approach proposed in this paper to address the problems discussed above is to utilize the variety of users' mobile and wired devices by clustering them. In the remainder, this cluster is referred to as the user's home swarm. The home swarm acts as a virtual node in a reciprocal incentive scheme. The main idea is to balance the load of reciprocation in a user's home swarm, thus allowing consumption and donation of resources to happen on different devices. An example of a random graph with clustered home swarms is given in Figure 2a. Assuming node $B_{1}$ to be a home access point and node $B_{2}$ to be a mobile device connected via a $3 \mathrm{G}$ cellular link, the proposed idea is that $B_{1}$ contributes resources by uploading content, while $B_{2}$ consumes the same amount of resources without reciprocating itself.

A conceptual architecture to provide this functionality is depicted in Figure 2b. It includes the P2P overlay functionality, which commonly consist of 
two main building blocks, one performing Neighborhood Selection using a Peer Sampling Service (e.g. a tracker server), the other performing Chunk Scheduling. Both consult the Incentive Component and take its suggestions (usually a priority list of peers to serve next) into account, when deciding on whom to serve which chunk of a file. In the following, the Incentive Component is decomposed into its subcomponents.

The basic component of the proposed incentive architecture is the Home Swarm Clustering Service. This service relies on the Peer Sampling Service for rendezvous and provides fraud proof means to tell apart which peers belong to the same home swarm and which do not. Atop, the Home Swarm Load Balancing component assumes a secure home swarm and can thus rely on all peers in the home swarm cluster to be reliable and to act in the interest of the user. The component implements the load balancing algorithm, using the Inbound/Outbound Accounting components to keep track of the home swarm's internal and external expenditures. The Mobility Support component is incorporated in the load balancing algorithm and deals with the special properties of mobile clients. The mobility support component will identify varying bandwidth/link-loss of mobile clients and care for initial credit to be generated even in absence of the mobile peer, thus caring for the non-energy related issues discussed in Section 2 .

\section{Preliminary Conclusions and Future Work}

The contributions of this paper are twofold. Firstly, the problem of reciprocation on mobile clients is substantiated with measurements followed by a discussion. Secondly, the home swarm concept and a conceptual architecture are introduced to address the problem.

As a next step the proposed architecture will be detailed by clearly separating the building blocks via interfaces, and a prototype will be implemented to show that the proposed approach relieves the load of mobile devices with respect to energy consumption and bandwidth in $\mathrm{P} 2 \mathrm{P}$ content distribution. The concept also implies algorithmic challenges with respect to resource allocation, trust and the general feasibility of the scheme which will be addressed in future work.

\section{References}

1. Sandvine: Fall 2012 Global Internet Phenomena Report, http://www.sandvine.com/news/global_broadband_trends.asp (last access January 2013)

2. Abboud, O., Zinner, T., Pussep, K., et al.: On the Impact of Quality Adaptation in SVC-based P2P Video-on-Demand Systems. ACM Multimedia Systems (2011)

3. Su, X., Dhaliwal, S.K.: Incentive Mechanisms in P2P Media Streaming Systems. IEEE Internet Computing 14(5), 74-81 (2010)

4. Liu, Z., Yanming, S., Ross, K.W., et al.: LayerP2P: Using Layered Video Chunks in P2P Live Streaming. IEEE Transactions on Multimedia 11(7), 1340-1352 (2009)

5. Bocek, T., Hecht, F.V., Hausheer, D., et al.: CompactPSH: An Efficient Transitive TFT Incentive Scheme for Peer-to-Peer Networks. IEEE LCN (2009) 